\title{
PERFIL SOCIODEMOGRÁFICO, SOCIOECONÔMICO E ESPORTIVO DE TENISTAS INFANTOJUVENIS BRASILEIROS DE ELITE
}

\author{
SOCIOECONOMIC, SOCIO-DEMOGRAPHIC AND SPORTS PROFILES OF \\ BRAZILIAN ELITE JUNIOR TENNIS PLAYERS
}

\author{
PERFIL SOCIODEMOGRÁFICO, SOCIOECONÓMICO Y DEPORTIVO DE \\ TENISTAS INFANTOJUVENILES BRASILEÑOS DE ÉLITE
}

\author{
Alexandro Andrade*, Pedro de Orleans Casagrande*, \\ Guilherme Guimarães Bevilacqua*, Fabiano Souza Pereira*, \\ Julianne Fic Alves*, Ana Luiza Goya*, Danilo Reis Coimbra*
}

Palavras chave: Classe social.

Tênis.

Esportes.

Atletas.

Keywords:

Social class.

Tennis.

Sports.

Athletes.

Palabras clave: Clase social.

Tenis.

Deportes.

Atletas
Resumo: 0 estudo objetivou descrever o perfil sociodemográfico, socioeconômico e esportivo de tenistas infantojuvenis brasileiros competitivos de elite. Participaram 130 tenistas, sendo 102 homens e 28 mulheres. A maioria dos tenistas é natural das Regiões Sudeste e Sul e houve um predomínio de tenistas treinando nessas regiões. A maioria dos tenistas pertence à classe socioeconômica $\mathrm{A}$. Em média, os tenistas iniciaram no tênis aos oito anos e o início em competições de tênis foi aos 11 anos. Os tempos médios de prática e de prática competitiva dos tenistas foram de sete e quatro anos, respectivamente. Já foram finalistas em competição internacional $16 \%$ dos atletas e aproximadamente a metade já disputou finais em competições internacionais e nacionais. Concluiu-se que os tenistas investigados são oriundos das classes econômicas mais altas. Além disso, os tenistas apresentaram grande volume de treino e tempo de prática.

Abstract: The study aimed to describe the sociodemographic, socioeconomic, and sports profiles of Brazilian competitive elite junior tennis players. A total of 130 athletes participated, being 102 males and 28 females. Most of them come from Brazil's Southeast and South and train in those regions. Most belong to the " $\mathrm{A}$ " socioeconomic class. On average, they began practicing tennis at the age of eight and started competing at the age of 11. Their average practice and competition times were seven and four years respectively. Some $(16 \%)$ have been finalists in international competitions and nearly half have already played finals in national and international competitions. Those players were found to come from Brazil's highest economic classes. Moreover, they showed large amount of training and practice time.

Resumen: Resumen: El estudio describe el perfil sociodemográfico, socioeconómico y deportivo de tenistas infantojuveniles de élite brasileños. Participaron 130 tenistas, siendo 102 hombres y 28 mujeres. La mayoría de los tenistas es natural de las regiones Sudeste y Sur y entrenan en esas regiones. La mayoría pertenece a la clase socioeconómica A. En promedio, comenzaron a practicar tenis a los ocho años y comenzaron a participar en competiciones a los 11 años. Los tiempos de práctica y de práctica competitiva fueron de siete y cuatro años en promedio, respectivamente. Han sido finalistas en competiciones internacionales $16 \%$ de los atletas y alrededor de la mitad ha disputado finales en competiciones nacionales e internacionales. Se concluye que los tenistas investigados son oriundos de las clases económicas más altas. Además, presentaron gran volumen de entrenamiento y tiempo de práctica..
*Universidade do Estado de Santa Catarina. Florianópolis, SC, Brasil. E-mail:

alexandro.andrade.phd@gmail.com pedrocasagrande.professor@gmail.com guibevi_1@hotmail.com; feibs@ig.com.br; juliannefic@hotmail.com; ana.luizas@hotmail.com; daniloreiscoimbra@yahoo.com.br

Recebido em: 08-06-2017 Aprovado em: 31-10-2017

DOI: http://dx.doi.org/10.22456/1982-8918.74041 (c) (1) (3) Licence 


\section{INTRODUÇÃO}

O campo de investigação que envolve os fatores determinantes no desenvolvimento atlético para o desempenho de elite tem sido foco de diversas pesquisas na perspectiva das Ciências do Esporte (BROUWERS; SOTIRIADOU; DE BOSSCHER, 2015; CÔTE et al., 2006; CÔTE; VIERIMAA, 2014; SOTIRIADOU; SHILLBURY, 2009; TUCKER, COLLINS, 2012). Especificamente no tênis, pesquisas têm investigado diversos fatores que podem explicar o sucesso esportivo na elite profissional. 0 mês de nascimento, a idade de entrada no ranking internacional e as estatísticas durante uma partida são algumas das variáveis associadas ao sucesso no tênis (BANE; REID; MORGAN, 2014; GIACOMINI, 1999; REID; MCMURTRIE; CRESPO, 2010).

Em uma perspectiva mais integrada dos fatores determinantes, De Bosscher et al. (2003) analisaram uma vasta literatura publicada a respeito. Os autores classificaram as pesquisas que investigaram os fatores determinantes em três níveis: macro, meso e micro. 0 nível macro está relacionado ao estudo do contexto social e cultural em que os atletas vivem (por exemplo, população, política econômica, sistema cultural, geografia e variação climática). No nível meso são os fatores relacionados às políticas esportivas, programas e estratégias que podem influenciar o desempenho a longo prazo. Fatores de nível micro investigaram os fatores individuais (por exemplo, qualidades genéticas) e ambientais (por exemplo, treinadores, estrutura de treinamento, patrocínio). Ainda, Brouwers, Sotiariadou e De Bosscher (2015) elaboraram uma estrutura conceitual de pilares que levam ao sucesso esportivo internacional no tênis. Dentro destes pilares, possuir adequado suporte financeiro, treinadores qualificados, instalações de treinamento, entre outros, sustentam e determinam o sucesso de alguns países no tênis profissional.

Investigar os fatores sociodemográficos e socioeconômicos no esporte não é uma tarefa simples, mas a ausência de estudos com esse objetivo leva a uma falta de clareza do impacto desses fatores na modalidade (WHITE; MCTEER, 2012). De fato, fatores socioeconômicos e sociodemográficos têm relação direta com a prática do esporte em um país, podendo contribuir ou ser desfavorável para o sucesso internacional na modalidade (DE BOSSCHER et al., 2009; DE BOSSCHER; DE KNOP; HEYNDELS, 2003; WHITE; MCTEER, 2012). Aliados a esses fatores, o histórico esportivo, a carga de treinamento atual e as condições de estrutura esportiva podem ser determinantes e devem ser levados em consideração nas políticas de incentivo para um país que almeja ter sucesso internacional no esporte.

O estudo de tenistas infantojuvenis tem despertado interesse de pesquisadores de diferentes áreas das Ciências do Esporte em todo o mundo (GIMENO et al., 2013; GUILLOT et al., 2015; ZIEMANN et al., 2013), enquanto que, no Brasil, poucos estudos têm sido realizados (CORTELA et al., 2010; PACHARONI; MASSA, 2012). Um estudo que investigue o perfil sociodemográfico, socioeconômico e esportivo de tenistas infantojuvenis brasileiros é importante, tendo em vista a carência de estudos analisando esses aspectos. Além do mais, o estudo pode impactar de maneira significativa no desenvolvimento do esporte no país, tendo em vista que muitas destes tenistas poderão representar o país futuramente. Conhecer e discutir esses fatores dos futuros tenistas profissionais brasileiros poderá contribuir para 0 desenvolvimento desses tenistas e contribuir para a evolução da modalidade dentro do cenário mundial. 
Assim, o objetivo deste estudo foi descrever o perfil sociodemográfico, socioeconômico e esportivo de tenistas infantojuvenis brasileiros competitivos de elite.

\section{MÉTODO}

O presente estudo se caracteriza como uma pesquisa descritiva de campo com delineamento transversal.

\subsection{Participantes}

Os participantes foram selecionados de forma não probabilística intencional adotando o critério do voluntariado. Participaram do estudo 155 tenistas. Foram excluídos tenistas que apresentaram erros excessivos de preenchimento, ou grande número de questões em branco. A amostra final foi composta por 130 tenistas, sendo 102 do sexo masculino $(78,5 \%)$ e 28 do sexo feminino (21,5\%). A faixa etária variou entre 13 e 18 anos $(\bar{x}=15,12 \pm 1,31)$. De acordo com o estudo de Swann, Moran e Piggott (2015), esses participantes foram classificados como tenistas infantojuvenis brasileiros competitivos de elite. Essa denominação se refere a atletas que competem no nível mais alto do seu esporte, mas ainda não têm sucesso nesse nível.

\subsection{Procedimentos}

A presente pesquisa foi aprovada pelo Comitê de Ética e Pesquisa em Seres Humanos da Universidade do Estado de Santa Catarina - UDESC (parecer: 988.292).

O critério de seleção foi por amostragem não-probabilística por conveniência e acessibilidade. Assim, as coletas foram realizadas em duas competições internacionais (Copa Guga Kuerten, Copa Santa Catarina) organizadas pela Confederação Brasileira de Tênis (CBT). Ambas as competições foram selecionadas pois possibilitariam o acesso a um maior número de atletas e ocorreriam em locais próximos, diminuindo, assim, os custos da pesquisa. Além do mais, são duas competições importantes no calendário do Tênis, pois pontuam para o ranking no Circuito Sul-americano - COSAT e Circuito Mundial Junior - ITF e contam com a participação de atletas de destaque no cenário nacional e internacional. Finalmente, essa opção metodológica se deu pois o acesso aos atletas foi consentido oficialmente pela organização nas duas competições.

O contato com os tenistas ocorreu durante 0 sign in (momento de confirmação da presença realizado um dia antes da competição). Todos assinaram o Termo de Assentimento informando quanto aos objetivos e procedimentos da pesquisa, assim como os riscos e benefícios da sua participação, concordando livremente em participar e que poderiam abandonar o estudo a qualquer momento.

Os tenistas responderam ao questionário individualmente, em local confortável e reservado. Os procedimentos foram acompanhados por uma equipe de pesquisadores com experiência que se colocaram à disposição para dúvidas e esclarecimentos durante as coletas. O tempo médio de preenchimento foi de 20 minutos. 


\subsection{Instrumentos}

Para avaliar as características esportivas foi utilizado o Questionário de Caracterização Esportiva de Atletas de Alto Rendimento - Tênis de Campo (QCAAR- TC), que vem sendo utilizado em pesquisas no esporte (ANDRADE et al. 2016; BRANDT et al., 2014). Esse questionário foi elaborado especificamente para atender aos objetivos deste estudo, com questões abertas e fechadas. Posteriormente, as respostas dos atletas foram categorizadas, permitindo uma análise quantitativa dos dados, permitindo uma melhor interpretação descritiva dos resultados encontrados.

As categorias analisadas no presente estudo foram: (a) Caracterização do Participante (sexo, idade); (b) Caracterização Sociodemográfica (estado e região de naturalidade, estado e região de treinamento); (c) Histórico Esportivo (idade de início no tênis, idade de início em competições, tempo de prática, tempo de prática competitiva); (d) Treinamento Esportivo Atual (frequência e volume de treino semanal, períodos de treino); (e) Patrocínio e Equipe Multidisciplinar e (f) Desempenho Esportivo (ranking atual, melhor resultado em competições na carreira). 0 ranking atual dos tenistas foi acessado via plataforma online da Confederação Brasileira de Tênis (CBT, 2014).

Também foi analisada a classe socioeconômica, por meio do Critério Padrão de Classificação Econômica Brasil (ABEP, 2015). Esse instrumento tem como objetivo estimar a classe socioeconômica, considerando o poder de compra das pessoas e famílias urbanas. Possui questões quanto ao grau de instrução do chefe da família (pessoa que mais contribui financeiramente com as despesas do lar), número de itens que a pessoa possui no domicílio e acesso aos serviços públicos. A soma dos pontos obtidos em cada questão resulta em um escore total, que representa a classificação socioeconômica. As classes são, decrescentemente: A (45-100 pontos), B1 (38-44 pontos), B2 (29-37 pontos), C1 (23-28 pontos), C2 (17-22 pontos) e D-E (0-16 pontos).

Os resultados foram tratados por meio da estatística descritiva, utilizando-se de média e desvio padrão, frequência e percentual. Todas as análises foram realizadas no Statistical Package for the Social Sciences versão 20.0 para Windows (SPSS Inc., Chicago, IL, USA).

\section{RESULTADOS}

\subsection{Características sociodemográficas e classe socioeconômica}

a maioria dos tenistas infantojuvenis brasileiros competitivos de elite investigados são naturais de cidades situadas nas Regiões Sudeste (60,3\%) e Sul (27\%) do Brasil, sendo que os estados de São Paulo e Santa Catarina foram os mais representativos. Em relação ao local de treinamento, observaram-se resultados semelhantes, com um predomínio de tenistas treinando nas Regiões Sudeste $(57,0 \%)$ e Sul $(34,4 \%)$ do Brasil e em cidades situadas nos estados de São Paulo e Santa Catarina (Figura 1).

A maioria dos tenistas infantojuvenis de elite pertence à classe socioeconômica $A$ $(84,6 \%)$. Apenas $6,9 \%$ são da classe B1 e $5,4 \%$ da classe B2. Da classe $C 1$, foi verificado somente um atleta $(0,8 \%)$. 
Figura 1 - Região e estado de naturalidade e de treinamento de tenistas brasileiros infantojuvenis competitivos de elite.

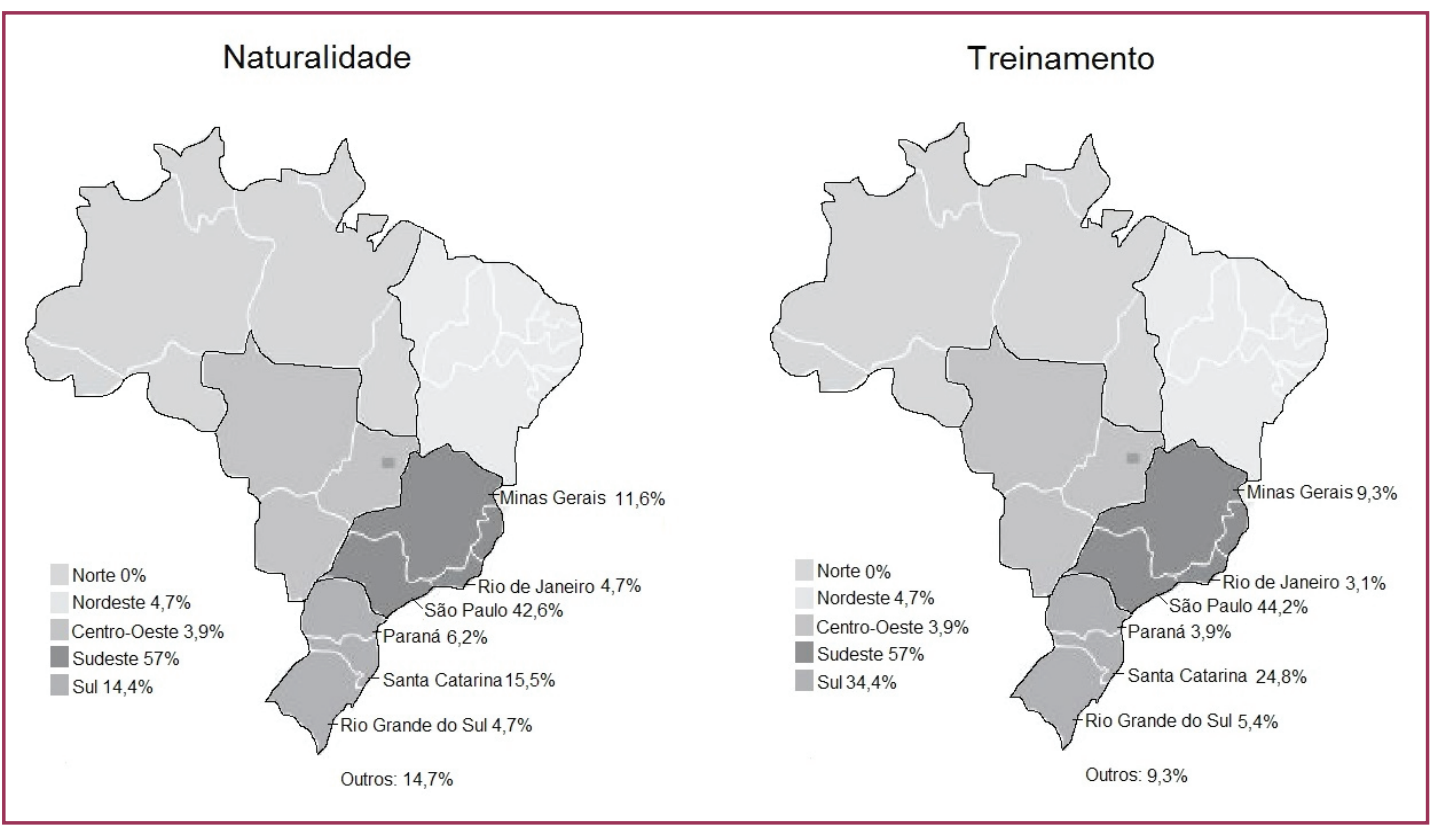

Fonte: Produzido pelos autores.

\subsection{Histórico esportivo}

Sobre as características do histórico esportivo dos tenistas infantojuvenis de elite investigados (Tabela 1), observou-se que, em média, eles iniciaram a prática do tênis aos oito anos de idade e o início em competições de tênis aos 11 anos de idade. 0 tempo médio de prática e o tempo de prática competitiva dos tenistas foi de sete anos e quatro anos, respectivamente.

Tabela 1 - Histórico esportivo dos tenistas infantojuvenis brasileiros competitivos de elite.

\begin{tabular}{lcc}
\hline Histórico Esportivo & $\overline{\mathbf{x}}$ & \pm \\
\hline Início no tênis (idade) & 8 & 2,5 \\
Início em competições (idade) & 11 & 2 \\
Tempo de prática (anos) & 7 & 2,5 \\
Tempo de prática competitiva (anos) & 4 & 2 \\
\hline
\end{tabular}

$\overline{\mathrm{X}}$ : Média; \pm : Desvio Padrão.

Fonte: Produzido pelos autores.

\subsection{Treinamento esportivo atual}

A maioria dos tenistas infantojuvenis de elite $(80 \%)$ treina cinco ou seis vezes por semana e nove tenistas treinam todos os dias da semana. Esses resultados demonstram a coerência entre o treinamento esportivo dos tenistas e o seu nível competitivo de elite (Tabela 2). O volume semanal de treino foi de $19( \pm 7)$ horas. Dos tenistas pesquisados, $20 \%$ treinam em dois períodos. 
Tabela 2 - Treinamento Esportivo Atual dos tenistas infantojuvenis brasileiros competitivos de elite.

\begin{tabular}{lccc}
\hline Treinamento Esportivo Atual & & Fr & $\%$ \\
\hline & $3 x$ & 4 & 3,0 \\
Frequência semanal & $4 x$ & 13 & 10,0 \\
& $5 x$ & 75 & 57,7 \\
& $6 x$ & 29 & 22,3 \\
Períodos de treino & $7 x$ & 9 & 7 \\
& Um & 104 & 80 \\
\hline
\end{tabular}

Fr: Frequência; \% Percentual válido.

Fonte: Produzido pelos autores.

\subsection{Patrocínio e equipe multidisciplinar}

Foi perguntado aos tenistas se recebem patrocínio e sobre a equipe de profissionais que auxiliam no treinamento. Dos 130 tenistas pesquisados, 65 (50,0\%) possuem algum tipo de patrocínio (financeiro, material esportivo ou para viagens). Observou-se que, dos 130 tenistas, $115(88,5 \%)$ têm treinador e preparador físico na equipe. Setenta e oito tenistas $(61,9 \%)$ contam com fisioterapeuta, 75 (59,5\%) nutricionista e 56 (44,4\%) psicólogo. Ressalta-se também que $38(30,2 \%)$ possuem uma equipe multidisciplinar composta por técnico, preparador físico, nutricionista, fisioterapeuta e psicólogo.

\subsection{Desempenho esportivo}

O desempenho esportivo dos tenistas foi avaliado através do melhor resultado na carreira e a posição no ranking atual. Destaca-se que $16 \%$ dos tenistas infantojuvenis de elite já foram finalistas em competição internacional e aproximadamente $50 \%$ já disputaram finais internacionais e nacionais. Dos 130 pesquisados, 114 (87,7\%) possuem ranking CBT, destes, 43,9\% estão posicionados entre os 100 melhores do ranking nacional. Destaca-se ainda que $27,1 \%$ dos tenistas estão posicionados entre os 50 melhores do ranking nacional (Tabela 3).

Tabela 3 - Desempenho Esportivo dos tenistas infantojuvenis brasileiros competitivos de elite.

\begin{tabular}{lllc}
\hline Desempenho Esportivo & & Fr & $\%$ \\
\hline \multirow{3}{*}{ Melhor resultado na carreira } & Finalista Internacional & 20 & 16,0 \\
& Finalista Nacional & 41 & 32,8 \\
& Finalista Estadual & 51 & 40,8 \\
& Outros Resultados & 13 & 10,4 \\
\hline \multirow{3}{*}{ Ranking atual } & Top 10 & 9 & 7,9 \\
& Top 20 & 11 & 9,6 \\
& Top 50 & 11 & 9,6 \\
& Top 100 & 19 & 16,7 \\
& Acima de 100 & 64 & 56,1 \\
\hline
\end{tabular}

Fr: Frequência; \% Percentual válido. Fonte: Produzido pelos autores. 


\section{DISCUSSÃO}

O objetivo deste estudo foi descrever e analisar o perfil sociodemográfico, socioeconômico e esportivo tenistas brasileiros infantojuvenis competitivos de elite.

\subsection{Perfil sociodemográfico e socioeconômico de tenistas brasileiros infantojuvenis competitivos de elite}

Ao descrever o perfil sociodemográfico, socioeconômico e esportivo de tenistas infantojuvenis brasileiros competitivos de elite, observamos que eles são naturais e estão treinando predominantemente nas Regiões Sul e Sudeste do Brasil, semelhante ao estudo de Cafruni, Marques e Gaya (2006), no qual os atletas também eram das Regiões Sul e Sudeste do Brasil. Ao cruzar esses resultados com o perfil econômico dos tenistas, é possível que exista uma relação entre o seu poder aquisitivo e o poder econômico dessas regiões, que parecem favorecer maiores e melhores condições para manter os tenistas treinando e competindo. Além disso, essas regiões estão entre as que mais organizam torneios infantojuvenis. Em consulta ao calendário esportivo da Confederação Brasileira de Tênis, dos 110 torneios infantojuvenis realizados no ano de 2014, 29,1\% ocorreram na Região Centro-Oeste, 24,5\% na Região Sul, 23,7\% na Região Sudeste, 15,4\% na Região Nordeste e 7,3\% na Região Norte.

Em relação à condição financeira dos tenistas, observou-se que a maioria $(84,6 \%)$ é da classe A e 51,6\% possui patrocínio. $O$ estudo de Casagrande et al. (2014) com tenistas infantojuvenis brasileiros mostrou que $72,7 \%$ dos atletas investigados não possuíam patrocínio, mas $70,5 \%$ se enquadravam nas classes econômicas mais altas. Estudos com tenistas profissionais brasileiros mostraram que esses tiveram dificuldades de sustentar financeiramente a carreira, sendo que muitos precisaram de ajuda familiar e externa, como, por exemplo, empréstimos em banco (PACHARONI; MASSA, 2012; PACHARONI; URSO; MASSA, 2014)

Em comparação a outros estudos com atletas brasileiros, o perfil socioeconômico dos tenistas foi semelhante ao de atletas de jiu-jítsu, mas divergente de estudos com atletas de natação e futebol. Andrade et al. (2014) apontaram que a maioria dos lutadores de jiu-jítsu pertencia à classe $\mathrm{A}$, enquanto que Fortes et al. (2012) relataram que os nadadores pertenciam à classe B. Já o estudo de Marques e Samulski (2009), apontou que 79,6\% dos atletas de futebol pertenciam à classe média ou baixa.

Estes achados apontam que Tênis, no Brasil, é um esporte elitizado, sendo praticado predominantemente por indivíduos da classe A. Assim, algumas questões relacionadas ao cenário do esporte no Brasil podem estar relacionadas a esse baixo número de praticantes nos estratos socioeconômicos mais baixos. Por exemplo, podemos destacar o número reduzido de quadras públicas para a prática da modalidade, 0 alto custo de materiais (ex.: raquetes, bolas e calçados específicos). Além disso, para iniciar no esporte, o tenista tem altos custos para aprender através de aulas individuais e coletivas, e no caso do esporte de alto rendimento o treinamento especializado é ainda mais caro. Ainda, os locais disponíveis para praticar o tênis são em clubes esportivos onde o indivíduo precisa se associar, e podem trazer custos adicionais de mensalidade.

Logo, políticas públicas de incentivo ao esporte devem ser realizadas através do fornecimento dos materiais esportivos, construção de quadras de acesso público gratuito, e o 
aumento no número de projetos sociais em comunidades visando à iniciação no tênis para os estratos socioeconômicos mais baixos, incentivando prática em nível recreacional e de saúde, bem como em nível competitivo.

\subsection{Histórico esportivo de tenistas infantojuvenis brasileiros competitivos de elite}

Os tenistas investigados apresentaram em média mais de seis anos de prática esportiva e quatro anos desde a primeira competição. A média de idade de início no esporte foi de oito anos e de inicio em competições de 11 anos. No estudo de Cafruni, Marques e Gaya (2006), apenas $25 \%$ dos atletas de diversas modalidades iniciaram no esporte antes dos 12 anos de idade. Segundo os autores, o início precoce na modalidade não significa que 0 atleta terá resultados importantes ao longo da carreira, demonstrando em seu estudo que os altos resultados obtidos no início da vida esportiva não se sustentaram na vida adulta. Brouwers, Bosscher e Sotiriadou (2012) afirmam que o bom desempenho de tenistas ainda quando crianças e adolescentes aumenta as chances de tornarem-se profissionais de elite, mas não é uma condição prévia para atingir o sucesso.

O desempenho dos tenistas foi avaliado através do ranking atual nacional da CBT e melhor desempenho em competições. Quase metade dos tenistas já atingiu o Top 20 do ranking nacional em algum momento de sua carreira e 48,8\% já foram campeões ou vice em competições internacionais ou nacionais. Um estudo que avaliou tenistas de vários países que alcançaram o Top 100 profissional observou que o melhor desempenho destes foi alcançado por volta dos 24 anos de idade, depois de em média 15 anos de prática esportiva (CORTELA et al., 2010). Também, dos tenistas que atingiram o Top 100 profissional, apenas 51,8\% estiveram classificados em algum momento da carreira no Top 20 Juniores Internacional (CORTELA et al., 2010).

Logo, deve haver um trabalho de orientação e conscientização de tenistas, pais, treinadores e demais envolvidos na carreira dos atletas para que as metas de desempenho sejam estabelecidas em longo prazo, respeitando as características biológicas individuais, 0 nível técnico e os interesses pessoais dos tenistas.

\subsection{Condições de treinamento de tenistas infantojuvenis brasileiros competitivos de elite}

Em relação a equipes de profissionais que trabalham com os tenistas, 30,2\% possuem equipe multidisciplinar com treinador, preparador físico, psicólogo, nutricionista e fisioterapeuta e boa parte tem outros profissionais auxiliando, além do treinador e preparador físico. Em estudo anterior com tenistas profissionais (PACHARONI; MASSA, 2012), quando questionados sobre o seu processo de formação esportiva, $80 \%$ consideraram que não tiveram treinamento planejado durante boa parte da sua formação, que só ocorreu posteriormente, com a melhora dos resultados.

Uma equipe multidisciplinar composta por profissionais capacitados pode implicar uma manifestação do potencial do atleta talentoso (COSTA; MASSA, 2009), tendo em vista que estes profissionais qualificados proporcionam melhores condições de treinamento ao atleta, através de planejamento e sistematização de programas para o desenvolvimento das 
habilidades (BERGAMO, 2004; COLANTONIO, 2007). Além disso, pode amenizar a carência, nessas regiões, de treinadores graduados em cursos voltados às Ciências do Esporte. Em estudo com 27 treinadores catarinenses de Tênis, Lima et al. (2014) relataram que 77,3\% são treinadores sem formação superior universitária em Educação Física. Do restante, 52,2\% têm nível superior em áreas não relacionadas com as Ciências do Esporte e apenas 22,7\% têm nível superior em Educação Física. Chiminazzo e Montagner (2009) ao investigarem 32 treinadores paulistas de Tênis relataram que 23 iniciaram um curso superior, mas apenas cinco em Educação Física e somente três eram formados.

No entanto, ainda que nossos resultados demonstrem tenistas treinando auxiliados por equipes multidisciplinares, futuramente devem ser analisados a frequência de atuação e 0 grau de envolvimento de fisioterapeutas, psicológicos e nutricionistas dentro do processo de treinamento.

\subsection{Treinamento esportivo atual de tenistas infantojuvenis brasileiros competitivos de elite}

Em relação ao treinamento atual, a maioria pratica o esporte de 5 a 6 vezes por semana e $20 \%$ treinam dois períodos, indicando que são tenistas com alto envolvimento no esporte. No estudo de De Francisco, Garce de los Fayos e Arce (2014) com atletas de diferentes modalidades esportivas, $24,3 \%$ treinam entre 7,5 e 12 horas, $6,6 \%$ entre 12,5 e 18 horas e 5,7\% treinam mais de 18 horas. Segundo alguns modelos de desenvolvimento de atleta, por volta dos 13 anos que o adolescente deve definir se especializar em seu esporte favorito ou continuar no esporte em nível recreacional, mas mesclando o esporte com outras atividades nessa fase (BANE; REID; MORGAN, 2014). No entanto, Segundo Côté e Vierimma (2014), estudos apontam que não é apropriado 0 adolescente se comprometer em treinamento intenso antes dos 16 anos, sendo que, após esse período, os atletas já se desenvolveram nos aspectos físico, cognitivo, social e emocional e possuem habilidades necessárias para o treinamento intenso e especializado. Para os tenistas que treinam dois períodos, o excesso de envolvimento no esporte não deve restringir o convívio social e atividades fora do esporte, tendo em vista que em longo prazo pode causar a síndrome de burnout (GUSTAFSSON; KENTTÄ; HASSMÉN, 2011), que se manifesta através de um esgotamento físico e emocional, desvalorização do esporte e um reduzido senso de realização esportiva, podendo levar a quedas de desempenho e abandono do esporte (GOODGER; WOLFENDEN; LAVALLEE, 2007; GOULD et al., 1996a; GOULD et al., 1996b). Novos estudos podem analisar as características de treinamento dos tenistas em função da idade e da especialização precoce.

\section{LIMITAÇÕES}

O presente estudo apresenta algumas limitações. Devido à escassez do estado da arte sobre a temática, no qual se observa uma carência de estudos com resultados e análises similares, a discussão dos resultados foi dificultada. Entretanto, o nosso apresenta dados inéditos, abrindo importantes questões que podem ser aprofundadas em futuras pesquisas investigando os aspectos socioeconômicos e demográficos em tenistas, possibilitando maior compreensão e discussão sobre esta temática pela comunidade científica. Ainda, nosso estudo discute questões como a necessidade de maior incentivo à prática do tênis por meio de políticas 
públicas que possibilitem que a experiência de praticar tênis seja proporcionada a indivíduos de todas as classes socioeconômicas.

\section{CONCLUSÕES}

Nossos resultados apresentam dados inéditos relacionados às características socioeconômicas, sociodemográficas e esportivas de tenistas infantojuvenis brasileiros competitivos de elite. Nossos resultados mostraram que os tenistas investigados são oriundos das classes econômicas mais altas e a maioria possui patrocínio. Ainda, a maioria dos tenistas investigados é natural de e treina em cidades situadas nas Regiões Sul e Sudeste do Brasil, que são as regiões com maior poder econômico. Quanto às características de treinamento, os tenistas estão altamente envolvidos no esporte, com grande volume de treino e tempo de prática. Além disso, no Brasil existem centros de treinamento onde os tenistas são auxiliados por uma equipe multidisciplinar, o que mostra um aumento nas condições de treinamento e estruturação de algumas equipes.

Esses dados são relevantes para treinadores, atletas, gestores esportivos e patrocinadores pois estão relacionados ao processo de formação de tenistas. No entanto, novas investigações devem ser realizadas a fim de aprofundar os pontos discutidos, ampliando o alcance amostral e as análises estatísticas inferenciais, como, por exemplo, qual o nível de apoio financeiro dos patrocinadores, grau de envolvimento e participação dos profissionais que auxiliam no treinamento das equipes multidisciplinares. Também deve aprofundar informações relacionadas ao fomento do tênis em diferentes regiões do país. Ademais, ressaltamos que nossos resultados não podem ser extrapolados para outras faixas etárias de tenistas e outras modalidades esportivas.

\section{REFERÊNCIAS}

ABEP. Associação Brasileira de Empresas de Pesquisa. Critério Padrão de Classificação Econômica Brasil. São Paulo, 2015. Disponível em: <http:// www.abep.org/Servicos/Download. aspx?id=09 >. Acesso em: 15 jul. 2014.

ANDRADE Alexandro et al. Mood and Performance: a Study of Elite Brazilian Volleyball Athletes. Journal of Sports Science and Medicine, v.15, n.4, p. 601-605, 2016.

ANDRADE, Alexandro et al. Tempo de reação, motivação e caracterização sociodemográfica de atletas iniciantes de jiu-jitsu. Revista Brasileira de Ciência e Movimento, v. 22, n. 1, p. 119129, 2014.

BANE, Michael Kenneth; REID, Machar; MORGAN, Stuart. Has player development in men's tennis really changed? An historical rankings perspective. Journal of Sports Sciences, v. 32, n. 15, p. 1477-1484, 2014.

BERGAMO, Vagner Roberto. Estabilidade: aspecto significativo na previsão do talento no basquetebol feminino. Revista Brasileira de Ciência e Movimento, v. 12, n. 2, p. 51-56, 2004. 
BRANDT, Ricardo et al. Mental health and associated factors in athletes during the open games of Santa Catarina. Revista Brasileira de Medicina do Esporte, v.20, n.4, p.276-280, 2014.

BROUWERS, Jessie; DE BOSSCHER, Veerle; SOTIRIADOU, Popi. An examination of the importance of performances in youth and junior competition as an indicator of later success in tennis. Sport Management Review, v. 15, n. 4, p. 461-475, 2012.

BROUWERS, Jessie; SOTIRIADOU Popi; DE BOSSCHER, Veerle. Sport-specific policies and factors that influence international success: The case of tennis. Sport Management Review, v. 18, n. 3, p. 343-358, 2015.

CAFRUNI, Cristina Borges; MARQUES, Antonio; GAYA, Adroaldo Cezar Araujo. Análise da carreira desportiva de atletas das regiões sul e sudeste do Brasil: Estudo dos resultados desportivos nas etapas de formação. Revista Portuguesa de Ciências do Desporto, v. 6, n. 1, p. 55-64, 2006.

CASAGRANDE, Pedro de Orleans et al. Burnout em tenistas brasileiros infanto-juvenis. Motrivivência, v. 10, n. 2, p. 60-71, 2014.

CHIMINAZZO, João Guilherme Cren; MONTAGNER, Paulo Cesar. Síndrome de burnout e esporte: a visão dos técnicos de tênis de campo. Journal of Physical Education UEM, v. 20, n. 2, p. 217-223, 2009.

COLANTONIO, Emilson. Detecção, seleção e promoção de talento esportivo: Considerações sobre a natação. Revista Brasileira de Ciência e Movimento, v. 15, n. 1, p. 127-135, 2007.

CONFEDERAÇÃO BRASILEIRA DE TÊNIS (CBT). Ranking brasileiro infanto-juvenil. Florianópolis, 2014. Disponível em: <www.tenisintegrado.com.br>. Acesso em: 20 nov. 2016.

CORTELA, Caio Correa. Tenistas top 100-um estudo sobre as idades de passagens pelos diferentes marcos da carreira desportiva. Pensar a Prática, v. 13, n. 3, p. 1-17, 2010.

COSTA, Juliana Miranda; MASSA, Marcelo. O processo de detecção e seleção de talentos no handebol. Revista Mackenzie de Educação Física e Esporte, v. 5, n. 2, p. 85-93, 2009.

CÔTÉ, Jean et al. When "where" is more important than "when": Birthplace and birthdate effects on the achievement of sporting expertise. Journal of Sports Sciences, v. 24, n. 10, p. 10651073, 2006.

CÔTÉ, Jean; VIERIMAA, Matthew. The developmental model of sport participation: 15 years after its first conceptualization. Science \& Sports, v. 29, n.1, p. 63-69, 2014.

DE BOSSCHER, Veerle et al. Explaining international sporting success: An international comparison of elite sport systems and policies in six countries. Sport Management Review, v. 12 , n. 3, p. 113-136, 2009.

DE BOSSCHER, Veerle; DE KNOP, Paul; HEYNDELS, Bruno. Comparing tennis success among countries. International Sports Studies, v. 25, n. 1, p. 49-68, 2003.

DE FRANCISCO, Cristina; GARCÉS DE LOS FAYOS, Enrique Javier; ARCE, Constantino. Burnout en deportistas: prevalencia del síndrome a través de dos medidas. Cuadernos de Psicología del Deporte, v. 14, n. 1, p. 29-38, 2014.

FORTES, Leonardo de Sousa et al. Insatisfação corporal e comportamento alimentar inadequado em jovens nadadores segundo níveis econômicos e competitivos. Jornal Brasileiro de Psiquiatria, v. 61, n. 1, p. 20-24, 2012. 
GIACOMINI, Craig. Association of birthdate with success of nationally ranked junior tennis players in the united states. Perceptual and Motor Skills, v. 89, n. 2, p. 381-386, 1999.

GIMENO, Fernando et al. Sportsmanship Rating Scale in Tennis Competition with Young Players. Procedia Social and Behavioral Sciences, v. 82, n.1, p. 740-744, 2013.

GOODGER, Kate; WOLFENDEN, Laura; LAVALLEE, David. Symptoms and consequences associated with three dimensions of burnout in junior tennis players. International Journal of Sport Psychology, v. 38, n. 4, p. 342-364, 2007.

GOULD, Daniel et al. Burnout in competitive junior tennis players: Qualitative Analysis. The Sport Psychologist, v. 10, n. 4, p. 341-366, 1996a.

GOULD, Daniel et al. Burnout in competitive junior tennis players: Individual differences in the Burnout experience. The Sport Psychologist, v. 11, n. 3, p. 257-276, $1996 \mathrm{~b}$.

GUILLOT, Aymeric et al. Implementation of Motor Imagery during Specific Aerobic Training Session in Young Tennis Players. Plos One, v. 10, n. 11, e0143331, nov. 2015. Disponível em: $<$ http://citeseer.ist.psu.edu/viewdoc/d;jsessionid=B2F79B557D5F63997A9CF2B6E4A458B8?doi=10.1 1.812.902\&rep=rep1\&type=pdf>. Acesso em: Acesso em: 15 jul. 2016.

GUSTAFSSON, Henrik; KENTTÄ, Göran; HASSMÉN, Peter. Athlete burnout: An integrated model and future research directions. International Review of Sport and Exercise Psychology, v. 4, n. 1, p. 23-24, 2011.

LIMA, Marcelo Bittencourt Neiva de et al. Perfil da formação inicial e permanente de treinadores de tênis de alto rendimento do Brasil. Pensar a Prática, v. 17, n. 1, p. 1-18, 2014.

MARQUES, Mauricio Pimenta; SAMULSKI, Dietmar Martin. Análise da carreira esportiva de jovens atletas de futebol na transição da fase amadora para a fase profissional: escolaridade, iniciação, contexto sócio-familiar e planejamento da carreira. Revista Brasileira de Educação Física e Esporte, v. 23, n. 2, p. 103-119, 2009.

PACHARONI, Rafael; MASSA, Marcelo. Processo de formação de tenistas talentosos. Motriz: Revista de Educação Física, v. 18, n. 2, p. 253-261, 2012.

PACHARONI, Rafael; URSO, Rodrigo Poles; MASSA, Marcelo. Condições limitantes sociais: 0 aporte financeiro e sua interferência no sucesso de tenistas brasileiros. Educação Física em Revista, v. 8, n. 1, p. 17-22, 2014.

REID, Machar; MCMURTRIE, Darren; CRESPO Miguel. The relationship between match statistics and top 100 ranking in professional men's tennis. International Journal of Performance Analysis in Sport, v. 10, n. 2, p. 131-138, 2010.

SOTIRIADOU, Kalliopi Popi; SHILBURY, David. Australian elite athlete development: An organisational perspective. Sport Management Review, v. 12, n. 3, p. 137-148, 2009.

SWANN, Christian; MORAN, Aidan; PIGGOTT, David. Defining elite athletes: Issues in the study of expert performance in sport psychology. Psychology of Sport and Exercise, v. 16, n. 1, p. 13-14, 2015.

TUCKER, Ross; COLLINS, Malcolm. What makes champions? A review of the relative contribution of genes and training to sporting success. British Journal of Sports Medicine, v. 46, n.8, p. 555-561, 2012. 
WHITE, Philip; MCTEER, William. Socioeconomic status and sport participation at different developmental stages during childhood and youth: Multivariate analyses using Canadian national survey data. Sociology of Sport Journal, v. 29, n. 2, p. 186-209, 2012.

ZIEMANN, Ewa et al. Exercise Training-Induced Changes in Inflammatory Mediators and Heat Shock Proteins in Young Tennis Players. Journal of Sports Science and Medicine, v. 12, n. 2, p. 282-289, 2013. 


\section{Apoio financeiro:}

Coordenação de Aperfeiçoamento de Pessoal de Nível Superior (CAPES), Fundação de Amparo à Pesquisa e Inovação do Estado de Santa Catarina (FAPESC). 